\title{
Support of Temporal Change Observation Using Augmented Reality for Learning
}

\author{
Takafumi Taketomi ${ }^{1}$, Angie Chen ${ }^{1}$, Goshiro Yamamoto ${ }^{1}$, and Hirokazu Kato ${ }^{1}$ \\ Nara Institute of Science and Technology, Japan \\ 8916-5 Takayama, Ikoma, Nara, Japan \\ E-mail: $\{$ takafumi-t, chen-a, goshiro, kato $\} @$ is.naist.jp
}

\begin{abstract}
An augmented reality (AR) technology enables to show an additional information by superimposing virtual objects onto the real world. The AR technology is gradually used in the learning environment for observing unseeable objects. Observation is the important process of inspecting a target object with significant details. It forms the basic of all scientific knowledge in education. However, there are only few AR applications which can visualize the temporal changes of the objects. In addition, the effect of this temporal change visualization by AR is not investigated from a scientific aspect. In this study, in order to clarify the effect of temporal change visualization by AR, we have compared the AR-based temporal change visualization method with the conventional temporal change visualization methods in the experiment. Especially, we set an observation of the plant growth as a practical scenario. Through the experiment, we have confirmed that superimpose the past appearance onto the user's viewpoint is effective for temporal change observation scenario.
\end{abstract}

Keywords: Augmented Reality, Temporal Change Visualization, Leaning Support

\section{Introduction}

Augmented Reality (AR) is a technology that integrates virtual elements into real environment that user can interact in real time. By the definition in the literature [1], AR has three characteristics: combines real and virtual, interactive in real time, and registered in 3-D. Due to the interaction, visualization and annotation features provided by $\mathrm{AR}$, many fields, such as entertainment, training, commercial, and education have been successfully implemented and explored. Especially, in the past decades, many AR applications for education have been developed and the usefulness of these applications were explored. However, the effectiveness of AR in the learning process is yet to be explored and evaluated based on learning theories. Furthermore, only a few existing AR prototype systems are created based on the theories to provide Augmented Reality Learning Experiences (ARLEs).

Experiential learning theory [4] proposed learning as a four-stage cycle and emphasizes the importance of experiences in the learning circle. Contextual learning, a curriculum design philosophy, concurs with the importance of experiential learning. It points out that learning only takes place when students process new information with 


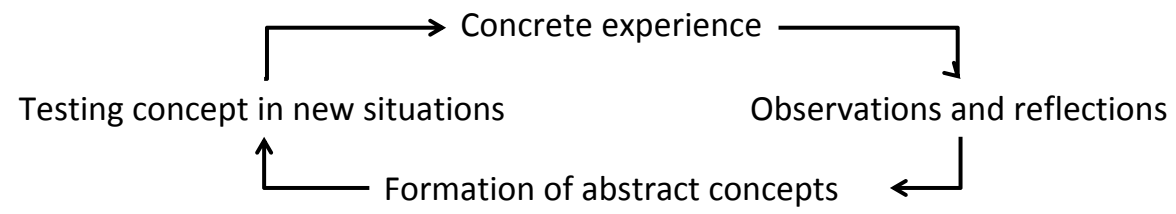

Fig. 1. The Experiential Learning Theory

their personal experiences [3]. The cycle of experiential learning theory (Figure 1) usually starts with having a concrete experience, followed by data collection from observation. Then, collected data is analyzed to make abstractions and generalizations, which are then tested on new situations. This testing stage starts the cycle again which gives the student another set of concrete experiences. In the traditional classroom learning, abstractions and formula are taught by teachers and textbooks. The stages of concrete experience and observations and reflections are limited. These two stages are usually carried out from field trips and experiments which can only be done in limited time and they cannot be repeated or accessed easily. On the other hand, AR can provide learning experiences to the user anytime with more flexible. This research focuses on observation not only because AR has the character as an display technology which is able to perform various visualization methods, but also because it is measurable. We can quantify the observation by evaluate how much information has been collected and if these information are better than information collected using non-AR method. We also noticed there are difficult scenarios in observation that observer needs assistance. We classify these difficult scenarios into three: limitation of senses, occlusion, and temporal changes. In these scenarios, the observation under temporal changes is less explored, and has less related works of supporting visual comparison. Overall, the goal of this research is to develop a prototype system that support observation under temporal changes in the classroom learning and evaluate the temporal change visualization methods.

\section{Observation Support by AR}

In the observation process, the observer collects data and information about an experience. However, there are several difficult observation scenarios in classroom learning. For example, to observe solar system planets or cells, to observe human organs and to observe physics collision. In this section, we briefly review the literatures related observation support by AR. These literatures can be classified into three groups by the aim of the applications: limitation of senses, occlusion, and temporal changes.

\subsection{Limitation of Senses}

Human has sense organs that are complicated structures which provides perception and sensation to the environment. However there are things which cannot be sensed due to the limitation of our senses. In many cases we can increase our sensory capabilities by 
using physical measuring devices. AR technology, with the characteristic of combines real and virtual and as a display technology, has been implemented to support visual difficulties or enhance vision. In recent years, it has been used as a tool to reduce the limitation by visualize subjects that are invisible to the naked eye.

The Real-time Visualization System [6] is an AR education tool combine traditional experiment and computer simulation. In traditional experiment, iron sand is used but it is time-consuming and impossible to deal with complicated models. Although these models can be handled by computer simulations, they are difficult for novice users, and provides little or no interactions. The Real-time Visualization System overcome these disadvantages which allows students to observe magnetic fields and move objects to change the field in real-time. Another example of such system [5] is used to teach organic chemistry by visualizing electron activity and dipole moment. In the traditional teaching, printed materials (e.g. graphics on paper or in books) or molecular models are used. The printed materials have various variety images but are limited to 2D pictures while molecular models have less variety but are display as a 3D structure. By using the system, students can chose elements from a booklet and the system will generate three-dimensional (3D) molecular models.

These systems support the observation under limitations of human senses and using AR to visualize the subjects and allow students to achieve observation in a approachable way. However, as we mentioned previously, these systems are not designed based on learning theories and did not conduct user experiments to evaluate the usefulness and effectiveness of their systems.

\subsection{Occlusion}

When the occlusion occurred, we need to physically obviate the blocking in order to make an observation. For example, in order to see the underground sewage system, we need to physically break the ground to see the actual pipes that are buried. AR technology as a display technology has been implemented to visualize the occlusion without the need to remove or obviate the blocking.

The mirracle [10] visuals the CT dataset for anatomy education. Using the system, the user can see the inside of the human anatomy without dissection courses that are often difficult to take place and requires a lot of effort. XRay-AR is a visualization method implemented in AR applications that shows a see-through affect [8]. Similar to the difficult scenario of limitation of senses in observation, the mirracle system used AR to visualize subjects that are hidden. By using the system, complicate experiment and troublesome process can be omit. However, they have the same drawbacks as well. The mirracle is not designed based on learning theories and no experiments were conducted; and XRay-AR have not been officially implemented to a particular educational AR prototype system.. Therefore, we cannot determine how learning is effected by the system or this technology.

\subsection{Temporal Changes}

Temporal changes mean that the changes of the subject happen over time. For example, the changes of height and wight of human being and the changes of colors of leaves 
according to seasons. To observe temporal changes, the observer needs to pay attention to the subjects carefully to notice the differences between statuses. Observe temporal changes is difficult to achieve because of the time factor. We are not able to see multiple statuses at the same time and compare the differences between statuses to know the changes. One of the most common methods of observing temporal changes is by visual comparison. Forsell et al. said "Visual comparison tasks take a central role in visual data exploration and analysis." [2] . In this paper, the authors also describe three phases of comparison:

1. Selection of pieces of information to be compared,

2. Arrangement of the pieces to suit the comparison, and

3. Carrying out the actual comparison.

By using AR, the first two phases are achieve automatically by the system. The Virtual Vouchers [7] is an example how AR assists visual comparison in non-classroom learning. In the field, when botanists need to identify a collected specimen or verify the existence of a new species, they initially consult their own personal knowledge and a paper field guide. In this case, the paper field guide might not contain full specimen collection or species samples, and it is difficult to use. However, the Virtual Vouchers system allows the user to access and view large amount of data and display the data side-by-side with physical specimens.

The CityViewAR ${ }^{1}$ is an other example for non-classroom learning of observing temporal changes using visual comparison. This system shows the street view before the 2011 earthquake in Christchurch onto the real buildings which are remained. In this case, students can compare the before and after scenes. The Campus Butterfly Ecology Learning System [11] presented a system that allows students to observer the virtual butterfly simulated and augmented in campus view. Different to the aforementioned systems which are field trip learning, the Campus Butterfly Ecology Learning System is used with the regular classes. However, this system only provides simulation but not visual comparison.

In this research, we focus on supporting temporal changes with the following reasons. First, this category is less explored, and has less related works of supporting visual comparison for temporal changes in classroom learning. Second, to support the observation of temporal changes, not only subjects and states are important but also how to "control" time needed to be taking care of. We propose that observation of temporal changes can benefit from AR technology and an pioneer evaluation is necessary to determine the effective in learning.

\section{Visualization of Temporal Changes by AR}

In this study, in order to visualize temporal changes of the object, we propose the viewmorphing based superimposition that displays the pass appearance of the object. Generally, in AR, superimposed objects are represented by 3D models. However, it is difficult to make a 3D model of the target object by novice users. Especially, in the learning environment, where the typical users may be children. In order to avoid making 3D model

\footnotetext{
${ }_{1}$ www.hitlabnz.org/cityviewar
} 
$<$ Offline phase>
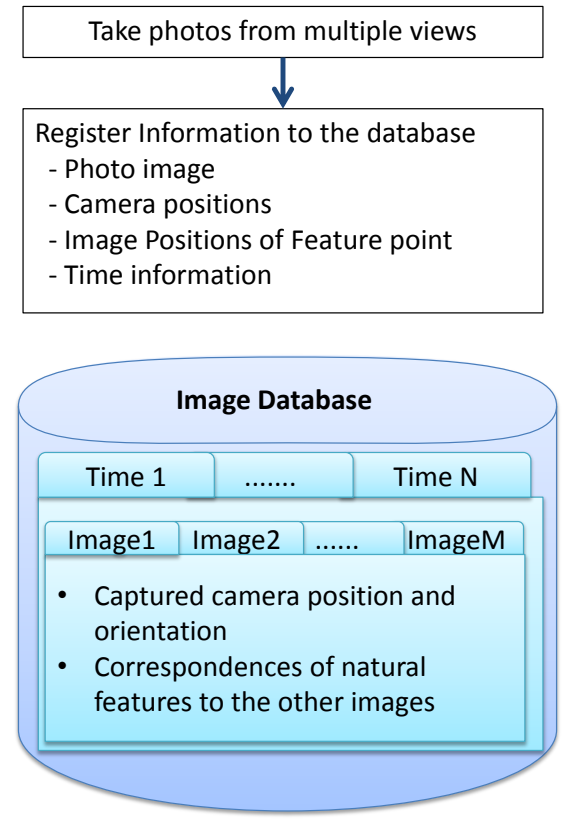

$<$ Online phase $>$

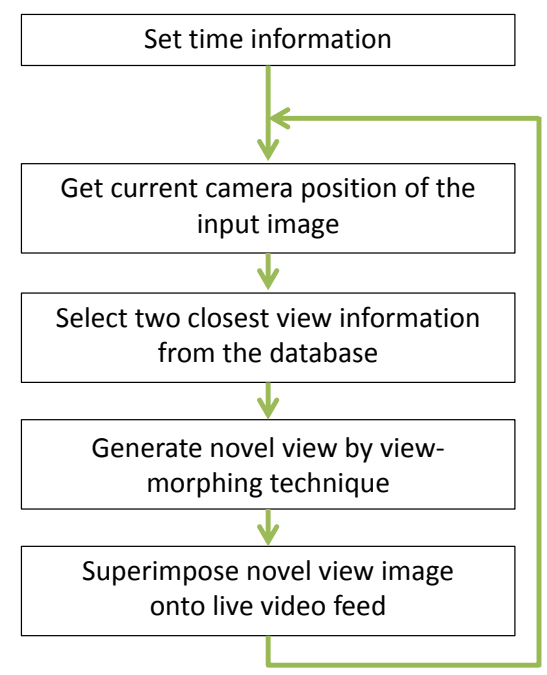

Fig. 2. The flow diagram of the proposed system.

of the target object, we employ the view-morphing method [9] to generate synthesized image. The view-morphing method can virtually generate arbitrary view image from a image pair. In order to realize the view-morphing based superimposition, the proposed method is composed of an offline image database construction process and an online novel view image generated and superimposition process as shown in Figure 2. In our method, we assume the environment, which fiducial markers are arranged around the target object and the relative position between the target object and fiducial markers is fixed. In this section, we describe the details of these processes.

\subsection{Construction of Image Database}

In the offline phase, the user is requested to take multiple photos of the target object from different camera positions and angles with temporal data of time t. The camera pose $C_{i}$ of the captured image $i$ is calculated using fiducial markers. In addition, feature points are extracted from the captured image, and then corresponding pairs of feature points between the captured image and images in the database are searched. Finally, for each photo, the camera pose $C_{i}$, image data, and corresponding information of natural features are registered to the database. In the registered image data, background information is removed by the simple background subtraction method using known background color information. 


\subsection{Visualization of Temporal Change by Novel View Generation}

In the online phase, firstly, the user manually select the target time for comparison, and then AR images are generated iteratively. In the AR view generation process, an image pair for novel view image generation is selected from the database with the following process.

1. Registered images are filtered to become candidate images based on the angels of optical axes and saved camera view direction.

2. Filter the candidate images using the distances between current camera position and registered positions.

3. Select two nearest camera positions that are located on the the left and right hand side of the current camera position.

After finishing the image selection process, the view-morphing process is executed. The original view-morphing method [9] is composed of pre-warping the image pairs, morphing the pre-warped images, and post-warping the morphed image. This method assumes to generate a novel view image without intrinsic and extrinsic camera parameters. From this assumption, the original method needs two image warping processes. On the other hand, in our implementation, camera poses and intrinsic camera parameters of the image pairs are known, and the camera pose and intrinsic camera parameters of the input image from live video feed are also known. By using these known information, we can simplify the original view-morphing method. In our method, the post-warping process is removed by generating the morphing image at the camera position of the input image as shown in Figure 3. The concrete view morphing process is follows.

1. Get the plane $\pi$ through three points: $C_{0}, C_{1}$, and $C_{s}$.

2. Derive the line $\overline{P C_{s}}$ which is the intersection between two planes: plane $\pi$ and plane $x=0$.

3. Get points $n_{0 i}$ and $n_{1 i}(i=0,1)$ that are corresponding points of the end points of the epipolar lines (projected by on line $\overline{P C_{s}}$ ) on $I_{0}$ and $I_{1}$.

4. Calculate the intersection range of $n_{0 i}$ and $n_{1 i}$ and the average point $m$ of this range.

5. Get point $C_{0}^{\prime}$ which is on the line-plane intersection of line $\overline{m C_{0}}$ and plane $z=0$, and point $C_{1}^{\prime}$ on the intersection of line of $\overline{m C_{1}}$ and plane $z=0$.

6. Project images $I_{0}$ and $I_{1}$ from $C_{0}$ and $C_{1}$ to $C_{0}^{\prime}$ and $C_{1}^{\prime}$.

$C_{0}, C_{1}$, and $C_{s}$ represents the camera positions of database image 0 , database image 1 , and input image, respectively. Finally, the generated novel view image is superimposed onto the input image as shown in Figure 4.

\section{Experiment}

We compared the effectiveness of our proposed visualization method for observation under temporal changes with other visualization methods through the use study. In this experiment, we set an observation of the plant growth as a practical scenario. 


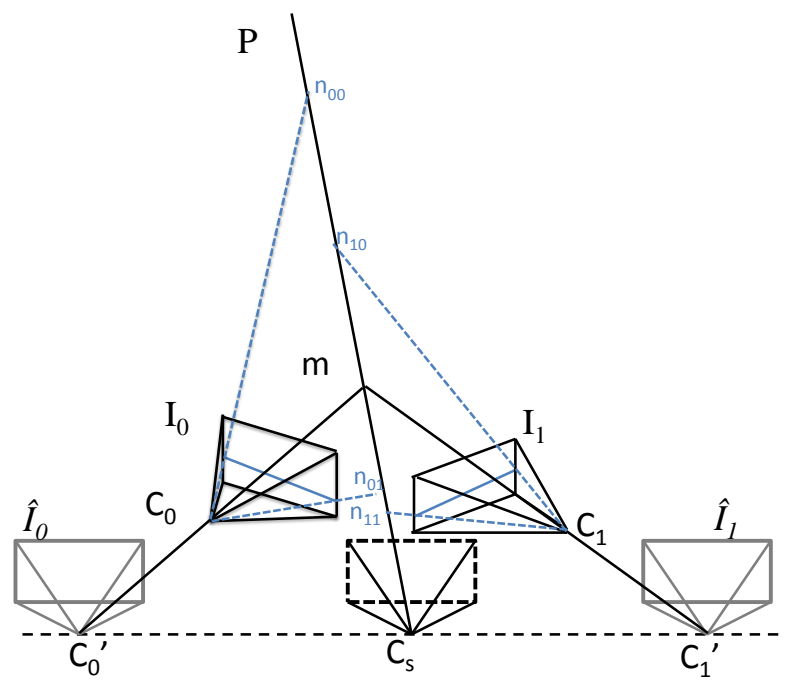

Fig. 3. View-morphing in the proposed method.
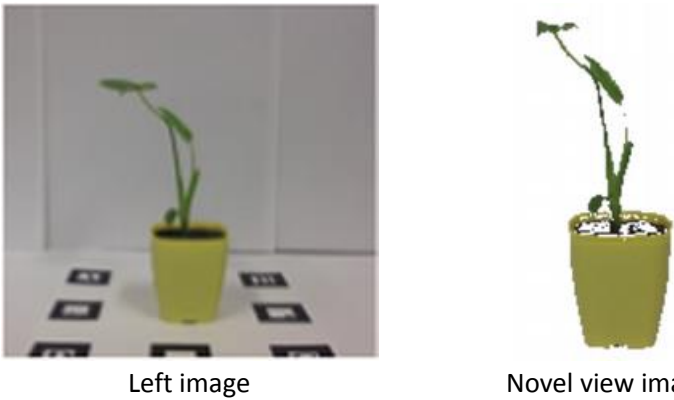

Novel view image

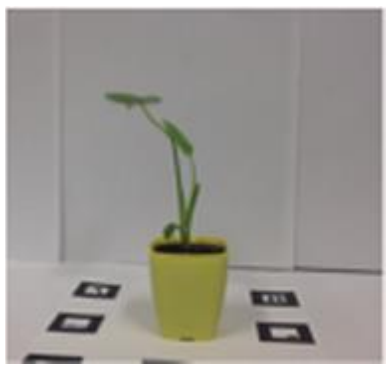

Right image

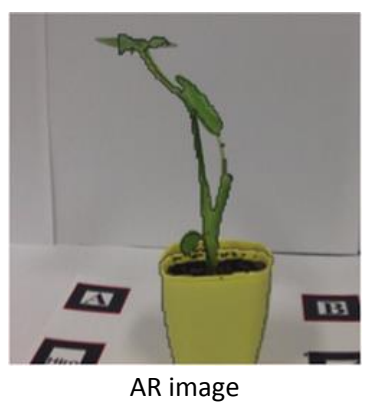

Fig. 4. Example of input and generated images. 


\subsection{Experimental Conditions}

Different types of visualization methods are suitable for different difficult scenarios of observation. Forsell et al. studied three approaches, side by side, shine-through, and fold, inspired by natural behaviors of printed paper [2]. In this experiment, different with paper-based comparison, we are not about to achieve the method of folding if we want to compare a physical 3-Dimension object. However, side by side and shinethrough are possible to accomplish by traditional methods as well as Augmented Reality technology. In this experiment, we carried out seven visualization methods under the category of side by side and shine-through (overlay) for observation and comparison as shown in Figure 5. Characteristics of each visualization method are described as followings.

\section{Side by side based visualization}

Printed images (Method A): Compare the images that were printed on papers with the subject by putting the papers beside the subject. Participants need to flip to the images that they wants to use for comparison.

Displayed images (Method B): Participants achieve comparison by locating the subject beside the computer screen where the images are displayed. Images displayed on computer screen are controlled using up and down arrows on the keyboard.

Displayed limited images on camera image (Method C): The system shows one of the registered images beside the orientation of the subject. In this condition, the system is without the view morphing function.. Participants may turn the subject around to observe from different angles.

Displayed novel view images on camera image (Method D): Participants are using the system with view morphing function for comparison. The system will generate in-between images based on the saved information and the current camera position.

\section{Overlay based visualization}

Printed transparent pictures (Method E): Compare images printed on transparency with the subject by putting the transparencies in the front of the subject. Comparison are carried out the subject and one image which is rendered besides it using AR system

AR with limited pictures (Method F): Compare the subject and the image rendered overlay on it using AR system.

AR with free viewpoint (Method G): Compare the subject and the novel view image generated by view-morphing overlay on it using AR system (the proposed system).

In the experiment, seven targets are provided to participants alternatively. 48 images were taken in 360 degrees around the target object with 7.5 degrees intervals were taken several days prior to the experiment and saved in database to represent the past status. The participants are required to use those images to conduct observation and comparison. 


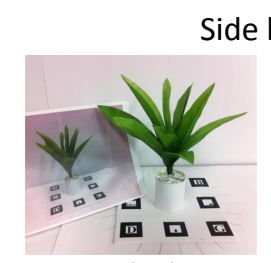

Method A

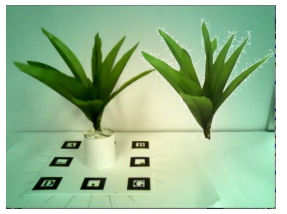

Method C
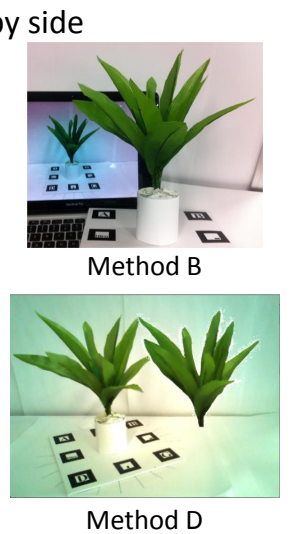

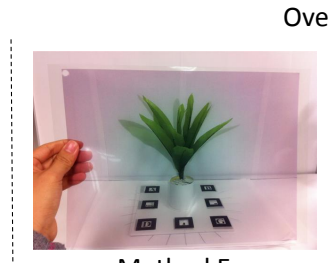

Method E

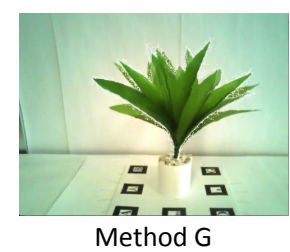

Overlay

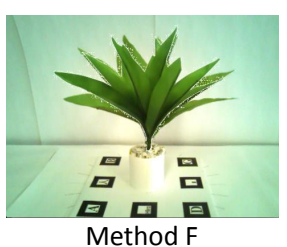

Method F

Fig. 5. Seven different visualization methods

\subsection{Design of the User Experiment}

In the experiment, the participant is required to observe the subject (plant), find the changes, and rank all seven methods based on the ease of observation and comparison after all the trials.. In each trial, the participant will received a set of images performed in different visualization methods and are requested to use these images to conduct observation and comparison.

The measurements include the quantity of information and the accuracy of information. The quantity is measured by how many changes can the participant notice. How many correct and incorrect identified changes are used for measuring the accuracy of the information. We also require the participant to answer about the advantages and disadvantages of each visualization method. The changes of these plants include new buds, leaves fallen, and changes of angles for outer leaves.

The procedure of the experiment is as follow. First of all, a brief interview with participants to gather basic information. This includes gender, age, any prior experience with AR applications. Secondly, explanations of the experiment, including the purpose of the experiment, the tasks for the participant, how the systems work, are provided. When the participant is ready, s/he can start to observe and compare using the target plant and provided visualization method. During the observation and comparison, the participants are required to mark the changes s/he found marking sheets. At the end of each visualization session, a short questioner which includes five self-report questions and section for comments of advantages and disadvantages about the visualization method. After all seven trials, the participants are required to rank all the visualization methods based on ease of observation and comparison. Lastly, the participants are asked to write the comments regarding to the visualization methods and the experiment. Overall, the experiment took about one and half hours including the post-experiment questionnaires. Five self-reported questions in the questionnaire of each visualization method trial session are listed below. $Q 1 \sim Q 5$ represent first to fifth questions. 
Q1 I think it is easy to notice the changes with this visualization method.

Q2 I think I found all the changes.

Q3 I think it is easy to see the changes of color.

Q4 I think it is easy to see the changes of height.

Q5 I think it is easy to see the changes of angle.

Answers of each question were given on a Liker Scale from 1 (Strongly disagree) to 5 (Strongly agree). The ranking scores of seven visualization methods are given from 7 (the best) to 1 (the worst).

\subsection{Result of the User Experiment}

The experiment involved 11 participants, 3 female and 8 male, with average age 29. Six of the participants do not have Augmented Reality (AR) development experience but participated AR-related experiments before. Meanwhile, the other five participants have AR development experiences. Each visualization method session took up to 6 minutes long and the whole experiment has a average duration of 90 minutes including the postexperiment questionnaire.

Table 1 shows the mean scores of questionnaires for each visualization method. For question 1, visualization method $\mathrm{F}$ has the highest mean score (3.73) and Method A has the lowest mean score of 1.91. Method $\mathrm{C}$ and method $\mathrm{G}$ share the highest mean score (3.00) in question 2. Methods C, F, G have the highest score for the ease of notice the changes of color, height, angle, respectively. In the mean score of ranking, visualization Method C (Side by side with non-view morphing) has the highest score (5.27), followed by visualization Method D (Side by side with view morphing AR application) with mean score 4.73. Visualization Method A (Side by side with printed images on papers) and B (Side by side with displayed images on computer screen) share the lowest mean score (2.91).

\subsection{Discussion}

Table 1 shows that detecting color is easier using visualization Methods $\mathrm{C}$ and $\mathrm{D}$ while is more difficult using Method $\mathrm{A}$ and $\mathrm{E}$. The methods that were scored higher for noticing the changes of height are Method F and C and Method B and E are scored lower.

Table 1. The mean scores of questionnaires and the mean scores of ranking for each visualization method. Bold font indicates the highest scores in each question.

\begin{tabular}{|l|c|c|c|c|c|c|}
\hline & Q1 & Q2 & Q3 & Q4 & Q5 & Score of Ranking \\
\hline \hline Method A & 1.91 & 2.18 & 2.09 & 2.36 & 2.55 & 2.91 \\
\hline Method B & 2.45 & 2.45 & 2.73 & 1.91 & 2.82 & 2.91 \\
\hline Method C & 3.18 & $\mathbf{3 . 0 0}$ & $\mathbf{3 . 5 5}$ & 3.45 & 3.18 & $\mathbf{5 . 2 7}$ \\
\hline Method D & 2.91 & 2.55 & 2.91 & 3.00 & 3.00 & 4.73 \\
\hline Method E & 2.27 & 2.09 & 1.36 & 1.91 & 2.82 & 3.82 \\
\hline Method F & $\mathbf{3 . 7 3}$ & 2.91 & 2.18 & $\mathbf{3 . 5 5}$ & 3.55 & 3.91 \\
\hline Method G & 3.27 & 3.00 & 2.73 & 3.36 & $\mathbf{4 . 0 0}$ & 4.45 \\
\hline
\end{tabular}


The methods that are easier for noticing the changes of angle were Methods $\mathrm{C}, \mathrm{F}$ and $\mathrm{G}$ where the more difficult ones were Method A, B and E. According to the experiment user ranking results, Method C, D and G were scored higher and Method A and $\mathrm{B}$ scored the lowest. These scores were reflected in the scores of ease of observation (Q1) for each methods. Method C, F G also scored the highest where Method A and B scored the lowest. Combining these two results we have confirmed that users chose AR methods (C, D, F and G) over non-AR methods (A, B, E) for the ease of observation.

Table 2 shows the mean of detection accuracy of each method. Considering the accuracy of changes detection of each method, Method A has the highest accuracy of changes detection while Method E has the lowest accuracy rate. Even though Method A has the best accuracy, participants did not think it was easy to use for observation and comparison. The reason for this outcome might be that we are familiar to manipulate and compare paper materials in our daily life. However when compared to other visualization methods, it is considerably more time consuming, difficult to manipulate and requires the user to do everything manually. In addition, we can see that Method F has higher detection error than Method G. We conjecture that the result might be caused by the occlusion. Four of participants reported that while using method $\mathrm{F}$ for observation and comparison, the occlusion occurs and interrupted their comparison process. We think the occlusion effect is suppressed by the view morphing in Method G.

Throughout the experiment and results, we have noticed that systems with and without view morphing yielded very similar results. We believe this is because the participants were able to access as many as 48 images (every 7.5 degrees around the target object). These images did not differ much to the images created from view morphing since the change of angle was very small. However, the result of detection error of the system with view morphing shows the possibility of improvement of the observation.

\section{Conclusion}

In this research, we are able to identify the most effective visualization method for observation under temporal changes. The result of our experiment shown that all camera image based visualization methods which includes the AR-based visualization method have higher score than methods without camera images. In the future, the quality of the synthesized images and resolution of camera needed to be improved. The differences between the systems which has view morphing and without view morphing yielded similar results. This might caused by the amount of images that were provided to the participants which is more than usual cases. As the result the selected images may not

Table 2. The mean accuracy of changes detection of each method.

\begin{tabular}{|l|c|c|c|c|c|c|c|}
\hline & Method A & Method B & Method C & Method D & Method E & Method F & Method G \\
\hline \hline $\begin{array}{l}\text { Accuracy of } \\
\text { changes detection }\end{array}$ & $\mathbf{0 . 8 5}$ & 0.84 & 0.77 & 0.84 & 0.67 & 0.77 & 0.76 \\
\hline $\begin{array}{l}\text { Number of } \\
\text { detection error }\end{array}$ & 13 & 12 & 12 & 8 & 12 & $\mathbf{1 9}$ & 12 \\
\hline
\end{tabular}


differ very much to the view morphing images. We need to conduct additional experiment with reduced number of registered images for non-view morphing system which is more similar to actual comparison and further verify our assumption.

\section{References}

1. R. T. Azuma. A survey of augmented reality. Presence, 6(4):355-385, 1997.

2. C. Forsell C. Tominski and J. Johansson. Interaction support for visual comparison inspired by natural behavior. IEEE Transactions on Visualization and Computer Graphics, 18:27192728, 2012.

3. What is contextual learning? http://www.cord.org/contextuallearning-definition/. Accessed: October 2012.

4. David A Kolb. Experiential learning: Experience as the source of learning and development, volume 1. Prentice-Hall, Inc., Englewood Cliffs, N.J., 1984.

5. L. Winterthaler B. Voegtli M. Fjeld, D. Hobi and P. Juchli. Teaching electronegativity and dipole moment in a tui. In In Proceedings of IEEE International Conference on Advanced Learning Technologies, pages 792-794. IEEE, 2004.

6. S. Noguchi S. Matsutomo, T. Miyauchi and H. Yamashita. Real-time visualization system of magnetic field utilizing augmented reality technology for education. Magnetics, IEEE Transactions on, 48:531-534, 2012.

7. S. Feiner S. White and J. Kopylec. Virtual vouchers: Prototyping a mobile augmented reality user interface for botanical species identification. In In Proceedings of 3DUI 2006 (IEEE Symp. on 3D User Interfaces, pages 119-126, 2006.

8. C. Sandor, A. Cunningham, A. Dey, and V.-V. Mattila. An augmented reality x-ray system based on visual saliency. In In Proceedings of IEEE International Symposium on Mixed and Augmented Reality, pages 27-36, Seoul, Korea, October 2010.

9. S. M. Seitz and C. R. Dyer. View morphing. In In Proceedings of the 23rd Annual Conference on Computer Graphics and Interactive Techniques, SIGGRAPH '96, pages 21-30, New York, NY, USA, 1996. ACM.

10. C. Bichlmeier T. Blum, V. Kleeberger and N. Navab. mirracle: An augmented reality magic mirror system for anatomy education. In Sabine Coquillart, Steven Feiner, and Kiyoshi Kiyokawa, editors, In Proceedings of IEEE Virtual Reality, pages 115-116. IEEE, 2012.

11. W. Tarng and K. L. Ou. A study of campus butterfly ecology learning system based on augmented reality and mobile learning. In In Proceedings of IEEE Seventh International Conference on Wireless, Mobile and Ubiquitous Technology in Education, pages 62-66. IEEE, 2012. 